\title{
Metamorphic Moving Horizon Estimation
}

\author{
He Kong and Salah Sukkarieh
}

\begin{abstract}
This paper considers a practical scenario where a classical estimation method might have already been implemented on a certain platform when one tries to apply more advanced techniques such as moving horizon estimation (MHE). We are interested to utilize MHE to upgrade, rather than completely discard, the existing estimation technique. This immediately raises the question how one can improve the estimation performance gradually based on the pre-estimator. To this end, we propose a general methodology which incorporates the pre-estimator with a tuning parameter $\lambda \in[0,1]$ into the quadratic cost functions that are usually adopted in MHE. We examine the above idea in two standard MHE frameworks that have been proposed in the existing literature. For both frameworks, when $\lambda=0$, the proposed strategy exactly matches the existing classical estimator; when the value of $\lambda$ is increased, the proposed strategy exhibits a more aggressive normalized forgetting effect towards the old data, thereby increasing the estimation performance gradually.
\end{abstract}

Index Terms - Least-squares estimation; State estimation; Constrained estimation; Recursive estimation.

\section{INTRODUCTION}

MHE is a systematic framework to handle constraints in estimation [1]-[7]. By far, various forms of MHE have been proposed. For example, in [8]-[9], the cost is optimized over the initial state and the process noise sequence to minimize the data fitting error. Other frameworks estimate only the initial state [10]-[12]. The concept of limited memory filtering has also been adopted in finite impulse response (FIR) filtering [13]-[14]. MHE and FIR filters are similar in that both methods only use recent measurements within a time window. However, there are major differences between them. For example, while the information contained in the measurements outside the moving horizon is captured in the so-called arrival cost in MHE [5, pp. 32-40], such information is ignored in FIR filtering. A situation that one often encounters when trying to apply MHE is that some traditional estimators might have already been implemented. For example, there would be some forms of Kalman filters embedded in today's most GPS devices. Replacing the existing estimation methods and related software and hardware by MHE is often time consuming and costly, if possible. A similar situation is faced by control engineers and this has motivated works to combine the merits of predictive and linear methods [15]-[17]. Especially, in [16], a general framework has been proposed to gradually improve performance using predictive control, incorporating an existing linear controller.

This document is an extended version of a technical communique accepted and to appear in Automatica. This work was supported in part by the Australian Centre for Field Robotics and in part by the Faculty of Engineering and Information Technologies, University of Sydney, Australia. The authors are with Australian Centre for Field Robotics, The University of Sydney, NSW, 2006, Australia. Email: h.kong@acfr.usyd.edu.au; salah.sukkarieh@sydney.edu.au. Corresponding author: He Kong, Tel.: +61 291140626.
The question we consider in this paper is to propose a MHE framework to gradually improve the estimation performance based on a pre-estimator. As such, we borrow the concept that is originally proposed in [16] for the control case, and consolidate the idea in two MHE frameworks that have been proposed in the existing literature [8], [10]-[11]. For both frameworks, we propose a methodology that can gradually improve the estimation performance with MHE, incorporating an existing estimator. This is achieved by the introduction of cost functions parameterized by $\lambda \in[0,1]$. When $\lambda$ changes, optimizing the cost functions renders a new estimator, we thus term the framework metamorphid $\sqrt{1}$ MHE (MMHE). An advantage of the proposed technique is that it can upgrade an existing classical method using MHE, thereby obtaining the constraint handling capabilities of MHE and avoiding the trouble involved in a completely new design of the estimator. A disadvantage of the framework, compared to classical estimation techniques, is that one has to solve an optimization problem at each sampling instant.

Notation: $\left[a_{1}, \cdots, a_{n}\right]$ denotes $\left[a_{1}^{\mathrm{T}} \cdots a_{n}^{\mathrm{T}}\right]^{\mathrm{T}}$, where $a_{1}, \cdots, a_{n}$ are scalars, vectors or matrices of proper dimensions; $\mathscr{I}_{i}^{j}$ denotes the set of integers between $i$ and $j$; a set $\mathscr{U} \subset \mathbf{R}^{n}$ is a $\mathscr{C}$-set if it is a compact, convex set that contains the origin in its (non-empty) interior; $\operatorname{diag}\left(M_{1}, \cdots, M_{s}\right)$ denotes a block diagonal matrix with $M_{1}, \cdots, M_{s}$ as its block diagonal entries, and $\operatorname{diag}_{N}(\cdot)$ denotes a block diagonal matrix with $N$ blocks. $\mathbf{1}_{n}$ denotes a $n$-dimensional column vector with all its elements as 1 .

\section{Metamorphic MHE}

The MHE framework in [8] considers the system

$$
x_{k+1}=A x_{k}+G w_{k}, y_{k}=C x_{k}+\nu_{k}
$$

where, $x_{k} \in \mathcal{X} \subset \mathbf{R}^{n}, w_{k} \in \mathcal{W} \subset \mathbf{R}^{m}$ and $\nu_{k} \in \mathcal{V} \subset \mathbf{R}^{p}$, respectively; the pair $(A, C)$ is assumed to be observable; the set $\mathcal{X}$ is compact and convex; $\mathcal{W}$ and $\mathcal{V}$ are both $\mathscr{C}$-sets. The variables $\left(x_{k}, w_{k}, y_{k}, \nu_{k}\right)$ in (1) represent the parameters of the real process. In an optimization-based estimation problem, they have corresponding decision variables and optimal decision variables, which we denote as $\left(\chi_{k}, \omega_{k}, \eta_{k}, v_{k}\right)$ and $\left(\widehat{x}_{k}, \widehat{w}_{k}, \widehat{y}_{k}, \widehat{\nu}_{k}\right)$, respectively. The MHE is a quadratic program (QP) in the form of

$$
\mathcal{M}_{T}:\left\{\begin{array}{c}
\min _{\chi_{T-N}, \omega_{T-N}^{T-1}} \tilde{\phi}_{T} \text { s.t. } \chi_{k} \in \mathcal{X}, k \in \mathscr{I}_{T-N}^{T} \\
\omega_{k} \in \mathcal{W}, v_{k} \in \mathcal{V}, k \in \mathscr{I}_{T-N}^{T-1}
\end{array},\right.
$$

with

$$
\widetilde{\phi}_{T}=\Theta_{T-N}\left(\chi_{T-N}\right)+\sum_{k=T-N}^{T-1}\left[v_{k}^{\mathrm{T}} R^{-1} v_{k}+\omega_{k}^{\mathrm{T}} Q^{-1} \omega_{k}\right],
$$

\footnotetext{
${ }^{1}$ As noted in [16], metamorphism is the recrystallization of pre-existing rocks due to physical/chemical changes.
} 
where, $\Theta_{T-N}\left(\chi_{T-N}\right)=\left(\chi_{T-N}-\widehat{x}_{T-N}^{m}\right) \Pi_{T-N}^{-1}\left(\chi_{T-N}-\right.$ $\left.\widehat{x}_{T-N}^{m}\right)+\widetilde{\phi}_{T-N}^{*}, \omega_{T-N}^{T-1}=\left\{\omega_{i}\right\}_{i=T-N}^{T-1}, \chi_{k}=\chi(k-(T-$ $\left.N) ; \chi_{T-N}, \omega_{T-N}^{k-1}\right), v_{k}=y_{k}-C \chi_{k}$; the matrix $\Pi_{T-N}$ is the solution to the ARE

$$
\Pi_{t}=G Q G^{\mathrm{T}}+A \Pi_{t-1} A^{\mathrm{T}}-A \Pi_{t-1} R_{t} \Pi_{t-1} A^{\mathrm{T}},
$$

with

$$
R_{t}=C^{\mathrm{T}}\left(R+C \Pi_{t-1} C^{\mathrm{T}}\right)^{-1} C,
$$

subject to the initial condition $\Pi_{0} ; \widehat{x}_{T-N}^{m}$ is the optimal rolling horizon state prediction at time $T-N$, i.e., $\widehat{x}_{T-N}^{m}=$ $\widehat{x}_{T-N \mid T-N-1}^{m}$, and $\widetilde{\phi}_{T-N}^{*}$ is the optimal cost of (2) at time $T-N$.

\section{A. Embellishing a pre-estimator into $M H E$}

Assume that for (1), we have the following Luenberger observer or stationary Kalman filter

$$
\widetilde{x}_{k+1}=A \widetilde{x}_{k}+L\left(y_{k}-\widetilde{y}_{k}\right), \widetilde{y}_{k}=C \widetilde{x}_{k},
$$

where, $L$ is chosen such that $A_{L}=A-L C$ is Schur stable. Define $e_{k+1}=x_{k+1}-\widetilde{x}_{k+1}$. Then it holds that

$$
e_{k+1}=A_{L} e_{k}+\vartheta_{k}
$$

with

$$
\vartheta_{k}=G w_{k}-L \nu_{k} \in \mathcal{Q}=G \mathcal{W} \ominus L \mathcal{V}
$$

Note that $\mathcal{Q}$ is also a $\mathscr{C}$-set since both $\mathcal{W}$ and $\mathcal{V}$ are $\mathscr{C}$-sets. Given $\rho\left(A_{L}\right)<1$, there exists a robust positively invariant $\mathscr{C}$-set $\mathscr{E}$ satisfying $A_{L} \mathscr{E} \oplus \mathcal{Q} \subseteq \mathscr{E}$ for system (5) (see [5], pp. 377). Define $x_{k}^{e}=\left[\widetilde{x}_{k}, e_{k}\right]$ and $\bar{w}_{k}=\left[w_{k}, v_{k}\right]$. From (1), (4), and (5), we have the augmented system

$$
x_{k+1}^{e}=A_{e} x_{k}^{e}+G_{e} \bar{w}_{k}, y_{k}=C_{e} x_{k}^{e}+\nu_{k},
$$

where,

$$
\begin{aligned}
A_{e} & =\left[\begin{array}{cc}
A & L C \\
0 & A_{L}
\end{array}\right], G_{e}=\left[\begin{array}{cc}
0 & L \\
G & -L
\end{array}\right] \\
C_{e} & =\left[\begin{array}{ll}
C & C
\end{array}\right]
\end{aligned}
$$

For (6), we have $x_{k}^{e} \in \overline{\mathcal{X}}, \bar{w}_{k} \in \overline{\mathcal{W}}$, where $\overline{\mathcal{X}}=\mathcal{X} \times \mathscr{E}$, $\overline{\mathcal{W}}=\mathcal{W} \times \mathcal{V}$. The variables $\left(x_{k}^{e}, \bar{w}_{k}, y_{k}, \nu_{k}\right)$ in (6) represent the parameters of the real augmented process, and we denote $\left(\chi_{k}^{e}, \bar{\omega}_{k}, \bar{\eta}_{k}, v_{k}\right)$ and $\left(\widehat{x}_{k}^{e}, \widehat{\bar{w}}_{k}, \widehat{y}_{k}, \widehat{\nu}_{k}\right)$ as the corresponding decision variables and the optimal solutions in the optimization, respectively. For notational ease, we still use $\widehat{y}_{k}$ and $\widehat{\nu}_{k}$ to denote the optimal output prediction and fitting error for (6) as for (1). For system (6), consider the constrained estimation problem

$$
\overline{\mathcal{M}}_{T}:\left\{\begin{array}{c}
\min _{\chi_{T-N}^{e}, \bar{\omega}_{T-1}^{T-1}} \vec{\phi}_{T} \text { s.t. } \chi_{k}^{e} \in \overline{\mathcal{X}}, k \in \mathscr{I}_{T-N}^{T} \\
\bar{\omega}_{k} \in \overline{\mathcal{W}}, v_{k} \in \mathcal{V}, k \in \mathscr{I}_{T-N}^{T-1}
\end{array}\right.
$$

where, $\chi_{k}^{e}=\chi^{e}\left(k ; \chi_{T-N}^{e}, \bar{\omega}_{T-N}^{k-1}\right), v_{k}=y_{k}-C \chi_{k}^{e}, \lambda \in[0,1]$, $\bar{\omega}_{T-N}^{T-1}=\left\{\bar{\omega}_{i}\right\}_{i=T-N}^{T-1}$

$$
\begin{aligned}
& \vec{\phi}_{T}=\lambda\left(\chi_{T-N}^{e}-\widehat{x}_{T-N}^{e m}\right) \Phi_{T-N}^{-1}\left(\chi_{T-N}^{e}-\widehat{x}_{T-N}^{e m}\right)+\lambda \vec{\phi}_{T-N}^{*} \\
& +\sum_{k=T-N}^{T-1}\left[(1-\lambda) \bar{\omega}_{k}^{\mathrm{T}} M \bar{\omega}_{k}+\lambda\left(v_{k}^{\mathrm{T}} R^{-1} v_{k}+\omega_{k}^{\mathrm{T}} Q^{-1} \omega_{k}\right)\right],
\end{aligned}
$$

in which, $R, Q, M>0 ; \Phi_{T-N}$ is a positive definite matrix to be discussed in the sequel; $\vec{\phi}_{T-N}^{*}$ is the optimal cost of (7) at time $T-N$, and thus is a constant parameter and can be safely ignored in the optimization; $\widehat{x}_{T-N}^{e m}$ is the optimal moving horizon state prediction at time $T-N$, i.e., $\widehat{x}_{T-N}^{e m}=$ $\widehat{x}_{T-N \mid T-N-1}^{e m}$.

When $\lambda=0$, one has $\vec{\phi}_{T}=\sum_{k=T-N}^{T-1} \bar{\omega}_{k}^{\mathrm{T}} M \bar{\omega}_{k}$. Given $0 \in$ $\overline{\mathcal{W}}$, the optimal decision variables are $\widehat{\bar{w}}_{i}=0$, for $i \in \mathscr{I}_{T-N}^{T-1}$. In this case, the optimal decision variables $\left(\widehat{x}_{k}^{e}, \widehat{\bar{w}}_{k}, \widehat{y}_{k}, \widehat{\nu}_{k}\right)$ satisfy $\widehat{x}_{k+1}^{e}=A_{e} \widehat{x}_{k}^{e}, \widehat{y}_{k}=C_{e} \widehat{x}_{k}^{e}$, i.e., the strategy reduces to a deterministic observer with the same gain as the preestimator (4). When $\lambda=1$, one has

$$
\begin{aligned}
& \vec{\phi}_{T}=\left(\chi_{T-N}^{e}-\widehat{x}_{T-N}^{e m}\right) \Phi_{T-N}^{-1}\left(\chi_{T-N}^{e}-\widehat{x}_{T-N}^{e m}\right) \\
& +\sum_{k=T-N}^{T-1}\left[\bar{\omega}_{k}^{\mathrm{T}} \bar{Q} \bar{\omega}_{k}+v_{k}^{\mathrm{T}} R^{-1} v_{k}\right],
\end{aligned}
$$

with $\bar{Q}=\operatorname{diag}\left(Q^{-1}, 0\right) \geq 0$. This is not a well-posed case since positive definiteness is required for the weight on $\bar{\omega}_{k}$. Thus, we will only consider the cases of $\lambda \in(0,1)$. Dividing $\vec{\phi}_{T}$ by $\lambda$ gives:

$$
\begin{aligned}
& \bar{\phi}_{T}=\lambda^{-1} \vec{\phi}_{T}=\sum_{k=T-N}^{T-1}\left[\bar{\omega}_{k}^{\mathrm{T}} Q_{e}^{-1} \bar{\omega}_{k}+v_{k}^{\mathrm{T}} R^{-1} v_{k}\right] \\
& +\left(\chi_{T-N}^{e}-\widehat{x}_{T-N}^{e m}\right) \Phi_{T-N}^{-1}\left(\chi_{T-N}^{e}-\widehat{x}_{T-N}^{e m}\right)
\end{aligned}
$$

where

$$
Q_{e}^{-1}=\frac{1-\lambda}{\lambda} M+\operatorname{diag}\left(Q^{-1}, 0\right)>0
$$

given $\lambda \in(0,1)$ and $M>0$. Moreover, one can consider a constrained estimation problem replacing $\vec{\phi}_{T}$ in (7) with $\bar{\phi}_{T}$ (8). Doing so will not affect optimality or stability.

\section{B. Stability ingredients for metamorphic $M H E$}

Proposition 1: Assume that $Q, R, M>0,(A, C)$ is observable. For $\lambda \in(0,1)$, we have: (i) $\left(A_{e}, C_{e}\right)$ is observable; (ii) if $\left(A, G Q^{-1 / 2}\right)$ is controllable, then $\left(A_{e}, G_{e} Q_{e}^{-1 / 2}\right)$ is controllable, with $Q_{e}$ defined in (8).

Proof. (i): Note that the poles of $A_{e}-L_{1} C_{e}$ can be arbitrarily placed within the unit circle by $L_{1}=\left[\begin{array}{ll}L & 0\end{array}\right]^{\mathrm{T}}$. (ii): Given $\left(A, G Q^{-1 / 2}\right)$ is controllable, there exists a matrix $K$ so that the poles of $A+G Q^{-1 / 2} K$ can be placed anywhere in the unit circle. Given $Q_{e}^{-1}>0$, for $\lambda \in(0,1)$, there exists a unique $Q_{s}>0$ such that $Q_{s}^{2}=Q_{e}^{-1}$ [18] (pp. 101). Denote

$$
K_{e}=Q_{s}^{-1}\left[\begin{array}{cc}
0 & Q^{-1 / 2} K \\
-C & -C
\end{array}\right]
$$

It can be verified that the poles of $A_{e}+G_{e} Q_{e}^{-1 / 2} K_{e}$ can be arbitrarily placed within the unit circle by $K_{e}$.

When one replaces $\vec{\phi}_{T}$ in (7) with $\bar{\phi}_{T}$ (8), the associated ARE for system (6) is

$$
\Phi_{T}=G_{e} Q_{e} G_{e}^{\mathrm{T}}+A_{e} \Phi_{T-1} A_{e}^{\mathrm{T}}-A_{e} R_{e} A_{e}^{\mathrm{T}}
$$

with $\Phi_{0}$ as the initial condition,

$$
R_{e}=\Phi_{T-1} C_{e}^{\mathrm{T}}\left(R+C_{e} \Phi_{T-1} C_{e}^{\mathrm{T}}\right)^{-1} C_{e} \Phi_{T-1},
$$


and $Q_{e}$ being defined in (8). Without constraints, one obtains the metamorphic Kalman filter

$$
\widehat{x}_{T}^{e}=A_{e} \widehat{x}_{T-1}^{e}+L_{e}\left(y_{T}-C_{e} A_{e} \widehat{x}_{T-1}^{e}\right),
$$

where,

$$
L_{e}=A_{e} \Phi_{T-1} C_{e}^{\mathrm{T}}\left(R+C_{e} \Phi_{T-1} C_{e}^{\mathrm{T}}\right)^{-1} .
$$

We have the following results regarding the invertibility of $\Phi_{T}$ (9) for later use. The results can be proved by following the ideas in [19], and thus the proof is skipped here.

Lemma 1: Assume that $Q, R, M, \Phi_{0}$ are positive definite, $(A, C)$ is observable. For $\lambda \in(0,1)$, we have $\Phi_{k}>0$, for all $k \geq 0$, if either of the following two conditions is satisfied: (i) $\left(A, G Q^{-1 / 2}\right)$ is controllable, and $\Phi_{0} \geq \Phi_{\infty}$; (ii) $G$ and $L$ are both nonsingular.

Theorem 1: Assume that $\Phi_{0}$ is chosen independently of $\lambda$, and $\Phi_{T}$ is updated according to the ARE (9). Suppose either of the two conditions in Lemma 1 is satisfied, i.e., $\Phi_{k}>0$, for $k \geq 0$, then for $\lambda \in(0,1)$, we have $\frac{d \Phi_{k}}{d \lambda} \geq 0$.

Proof. We prove the above result by induction. Since $\Phi_{0}$ is independent of $\lambda$, for $k=0$, we have $\frac{d \Phi_{0}}{d \lambda}=0$. When $k=1$, from (9), we have

$$
\begin{aligned}
& \Phi_{1}=G_{e} Q_{e} G_{e}^{\mathrm{T}}+A_{e} \Phi_{0} A_{e}^{\mathrm{T}} \\
& -A_{e} \Phi_{0} C_{e}^{\mathrm{T}}\left(R+C_{e} \Phi_{0} C_{e}^{\mathrm{T}}\right)^{-1} C_{e} \Phi_{0} A_{e}^{\mathrm{T}} .
\end{aligned}
$$

Note that the second and third items on the right hand side of the above equality is independent of $\lambda$. Therefore, it holds that

$$
\frac{d \Phi_{1}}{d \lambda}=G_{e} \frac{d Q_{e}}{d \lambda} G_{e}^{\mathrm{T}}
$$

where we have used the rule

$$
\frac{d X Y}{d x}=\frac{d X}{d x} Y+X \frac{d Y}{d x}
$$

in which, $X$ and $Y$ are two matrices of compatible dimensions, and $x$ is a scalar. Note, for $Q_{e}^{-1}$, we have (15). Hence the following holds

$$
\frac{d Q_{e}}{d \lambda}=\frac{1}{\lambda^{2}} Q_{e} M Q_{e}>0 .
$$

By combing the above expression and (10), we have

$$
\frac{d \Phi_{1}}{d \lambda}=G_{e} \frac{d Q_{e}}{d \lambda} G_{e}^{\mathrm{T}}=\frac{1}{\lambda^{2}} G_{e} Q_{e} M Q_{e} G_{e}^{\mathrm{T}} \geq 0 .
$$

Now, for $k=2$, we have

$$
\begin{aligned}
& \frac{d \Phi_{2}}{d \lambda}=G_{e} \frac{d Q_{e}}{d \lambda} G_{e}^{\mathrm{T}}+A_{e} \frac{d \Phi_{1}}{d \lambda} A_{e}^{\mathrm{T}}-A_{e} \frac{d \Phi_{1}}{d \lambda} C_{e}^{\mathrm{T}} \bar{R}^{-1} C_{e} \Phi_{1} \bar{A}^{\mathrm{T}} \\
& -A_{e} \Phi_{1} C_{e}^{\mathrm{T}} \frac{d \bar{R}-1}{d \lambda} C_{e} \Phi_{1} A_{e}^{\mathrm{T}}-A_{e} \Phi_{1} C_{e}^{\mathrm{T}} \frac{\bar{R}^{-1}}{C_{e} \frac{d \Phi_{1}}{d \lambda} A_{e}^{\mathrm{T}}} \\
& =G_{e} \frac{d Q_{e}}{d \lambda} G_{e}^{\mathrm{T}}+A_{e}\left(\frac{d \Phi_{1}}{d \lambda}-\frac{d \Phi_{1}}{d \lambda} C_{e}^{\mathrm{T}} \bar{R}{ }^{-1} C_{e} \Phi_{1}\right. \\
& \left.-\Phi_{1} C_{e}^{\mathrm{T}} \frac{d \bar{R}^{-1}}{d \lambda} C_{e} \Phi_{1} \bar{A}^{\mathrm{T}}-\Phi_{1} C_{e}^{\mathrm{T}} \bar{R}^{-1} C_{e} \frac{d \Phi_{1}}{d \lambda}\right) A_{e}^{\mathrm{T}},
\end{aligned}
$$

where, $\bar{R}=R+C_{e} \Phi_{1} C_{e}^{\mathrm{T}}$. Since $\frac{d \Phi_{1}}{d \lambda} \geq 0$, there exists a unique $\Delta \geq 0$ such that $\Delta^{2}=\frac{d \Phi_{1}}{d \lambda}[18]$ (pp. 101). Based on the expression of $\frac{d \Phi_{1}}{d \lambda}, 111$, and the fact that

$$
\frac{d X^{-1}}{d x}=-X^{-1} \frac{d X}{d x} X^{-1}
$$

in which, $X$ is a nonsingular matrix with its components as functions of a scalar $x$, one has

$$
\begin{aligned}
& \frac{d \bar{R}^{-1}}{d \lambda}=-\bar{R}^{-1} \frac{d\left(R+C_{e} \Phi_{1} C_{e}^{\mathrm{T}}\right)}{d \lambda} \bar{R}^{-1} \\
& \quad=-\bar{R}^{-1} C_{e} \Delta^{2} C_{e}^{\mathrm{T}} \bar{R}{ }^{-1} \leq 0 .
\end{aligned}
$$

Therefore, we have

$$
\begin{aligned}
& A_{e}\left(\frac{d \Phi_{1}}{d \lambda}-\frac{d \Phi_{1}}{d \lambda} C_{e}^{\mathrm{T}} \bar{R}^{-1} C_{e} \Phi_{1}\right. \\
& \left.-\Phi_{1} C_{e}^{\mathrm{T}} \frac{d \bar{R}^{-1}}{d \lambda} C_{e} \Phi_{1}-\Phi_{1} C_{e}^{\mathrm{T}} \bar{R}^{-1} C_{e} \frac{d \Phi_{1}}{d \lambda}\right) A_{e}^{\mathrm{T}} \\
& =A_{e}\left(\Delta^{2}-\Delta^{2} C_{e}^{\mathrm{T}} \bar{R}^{-1} C_{e} \Phi_{1}-\Phi_{1} \bar{C}^{\mathrm{T}} \bar{R}-1 \bar{C} \Delta^{2}\right. \\
& \left.+\Phi_{1} C_{e}^{\mathrm{T}} \bar{R}^{-1} C_{e} \Delta^{2} C_{e}^{\mathrm{T}} \bar{R}^{-1} C_{e} \Phi_{1}\right) A_{e}^{\mathrm{T}} \\
& =A_{e}\left(\Delta-\Phi_{1} C_{e}^{\mathrm{T}} \bar{R}^{-1} C_{e} \Delta\right)\left(\Delta-\Phi_{1} C_{e}^{\mathrm{T}} \bar{R}^{-1} C_{e} \Delta\right)^{\mathrm{T}} A_{e}^{\mathrm{T}} \geq 0 .
\end{aligned}
$$

Since $G_{e} \frac{d Q_{e}}{d \lambda} G_{e}^{\mathrm{T}} \geq 0$, from the expression of $\frac{d \Phi_{2}}{d \lambda}$ given after (12), we have $\frac{d \Phi_{2}}{d \lambda} \geq 0$. The above procedure can be carried out for $k \geq 3$. Thus, for $k \geq 0$ and $\lambda \in(0,1)$, it holds that $\frac{d \Phi_{k}}{d \lambda} \geq \overline{0}$.

When $\Phi_{0}>\Phi_{\infty}$, the assumption that $\Phi_{0}$ is chosen independently of $\lambda$ can be satisfied by selecting a sufficiently large $\Phi_{0}$. Therefore, the results in Theorem 1 can be applied for this case. When $\Phi_{0}=\Phi_{\infty}, \Phi_{k}=\Phi_{\infty}$, for all $k \geq 0$, e.g., $\Phi_{0}$ is dependent of $\lambda$, as $\Phi_{\infty}$ is. We have the following results complementary to Theorem 1 .

Corollary 1: Assume $Q, R, M$ are positive definite, $(A, C)$ and $\left(A, G Q^{-1 / 2}\right)$ are observable and controllable, respectively. Suppose $G$ and $L$ are nonsingular. If $\Phi_{0}=\Phi_{\infty}$, then for $\lambda \in(0,1)$, it holds that $\frac{d \Phi_{\infty}}{d \lambda}>0$.

Proof. Note that $\Phi_{\infty}$ satisfies

$$
\Phi_{\infty}=G_{e} Q_{e} G_{e}^{\mathrm{T}}+A_{e} \Phi_{\infty} A_{e}^{\mathrm{T}}-A_{e} \Phi_{\infty} C_{e}^{\mathrm{T}} \bar{R}_{\infty}^{-1} C_{e} \Phi_{\infty} A_{e}^{\mathrm{T}},
$$

where, $\bar{R}_{\infty}=R+C_{e} \Phi_{\infty} C_{e}^{\mathrm{T}}$. Denote $\bar{L}=A_{e} \Phi_{\infty} C_{e}^{\mathrm{T}} \bar{R}_{\infty}^{-1}$ and $\bar{A}_{L}=A_{e}-\bar{L} C_{e}$. Differentiating the above ARE w.r.t. $\lambda$ on both sides gives us

$$
\begin{aligned}
& \frac{d \Phi_{\infty}}{d \lambda}=G_{e} \frac{d Q_{e}}{d \lambda} G_{e}^{\mathrm{T}}+A_{e} \frac{d \Phi_{\infty}}{d \lambda} A_{e}^{\mathrm{T}}-A_{e} \frac{d \Phi_{\infty}}{d \lambda} C_{e}^{\mathrm{T}} \bar{R}_{\infty}^{-1} C_{e} \Phi_{\infty} A_{e}^{\mathrm{T}} \\
& -A_{e} \Phi_{\infty} C_{e}^{\mathrm{T}} \frac{d \bar{R}_{\infty}^{-1}}{d \lambda} C_{e} \Phi_{\infty} A_{e}^{\mathrm{T}}-A_{e} \Phi_{\infty} C_{e}^{\mathrm{T}} \bar{R}_{\infty}^{-1} C_{e} \frac{d \Phi_{\infty}}{d \lambda} A_{e}^{\mathrm{T}} \\
& =G_{e} \frac{d Q_{e}}{d \lambda} G_{e}^{\mathrm{T}}+\bar{A}_{L} \frac{d \Phi_{\infty}}{d \lambda} \bar{A}_{L}^{\mathrm{T}},
\end{aligned}
$$

where we have used (11) and (13). When $G$ and $L$ are nonsingular, one has that $G_{e}$ is full rank, e.g.,

$$
\bar{A}_{L} \frac{d \Phi_{\infty}}{d \lambda} \bar{A}_{L}^{\mathrm{T}}-\frac{d \Phi_{\infty}}{d \lambda}=-G_{e} \frac{d Q_{e}}{d \lambda} G_{e}^{\mathrm{T}}<0,
$$

given (12). If $(A, C)$ and $\left(A, G Q^{-1 / 2}\right)$ are observable and controllable, respectively, $\left(A_{e}, C_{e}\right)$ and $\left(A_{e}, G_{e} Q_{e}^{-1 / 2}\right)$ are observable and controllable, respectively, as proved in Proposition 1 Under such conditions, $\bar{A}_{L}$ is Schur stable (see, e.g., Theorem 4.1 in [20]). Therefore, the discrete-time Lyapunov matrix equation (14) has a unique positive definite solution, i.e., $\frac{d \Phi_{\infty}}{d \lambda}>0$.

We assume $R, M$ to be independent of $\lambda$. It then can be verified that

$$
\frac{d\left(Q_{e}^{-1}\right)}{d \lambda}=-\frac{1}{\lambda^{2}} M<0 .
$$

Differentiating (8) w.r.t. $\lambda$ gives

$$
\begin{aligned}
& \frac{d \bar{\phi}_{T}}{d \lambda}=-\frac{1}{\lambda^{2}} \sum_{k=0}^{T-1} \bar{\omega}_{k}^{\mathrm{T}} M \bar{\omega}_{k} \\
& -\left(\chi_{T-N}^{e}-\widehat{x}_{T-N}^{e m}\right)^{\mathrm{T}} \Phi_{T-N}^{-1} \frac{d \Phi_{T-N}}{d \lambda} \Phi_{T-N}^{-1}\left(\chi_{T-N}^{e}-\widehat{x}_{T-N}^{e m}\right),
\end{aligned}
$$


where fact (13) is used, in which, $X$ is a nonsingular matrix. If $\Phi_{0}$ is independent of $\lambda, \frac{d \Phi_{0}}{d \lambda}=0$, from Theorem 11, one has $\frac{d \Phi_{k}}{d \lambda} \geq 0$. If $\Phi_{0}$ is dependent of $\lambda$, e.g., $\Phi_{0}$ is chosen to be the steady-state solution of the ARE (9), it is established in Corollary 1 that $\frac{d \Phi_{\infty}}{d \lambda} \geq 0$. Note that for the above both cases, the inverse of matrix $\Phi_{k}$ or $\Phi_{\infty}$ is used in MMHE as the weighting on the state estimate obtained using the old measurement outside the moving horizon. In other words, an increase of $\lambda$ results in a more aggressive forgetting effect towards the estimate using old data. Therefore, we remark that the increase of $\lambda$ stands for the designers' willingness to rely on more recent, rather than relatively old, data. Based on the above results and by following the steps in [8], one can establish the stability of both metamorphic FIE and MHE in the sense of an observer. Due to limited space, details of the stability analysis are skipped here.

\section{Metamorphic MHE of the Initial State}

The MHE framework in [10]-[11] considers the system

$$
x_{k+1}=A x_{k}+B u_{k}+w_{k}, y_{k}=C x_{k}+\nu_{k}
$$

where, $x_{k} \in \mathbf{R}^{n}, w_{k} \in \mathcal{W} \subset \mathbf{R}^{n}$ and $\nu_{k} \in \mathcal{V} \subset \mathbf{R}^{p}$ with $\mathcal{W}$ and $\mathcal{V}$ standing for two $\mathscr{C}$-sets; $(A, C)$ is observable. At each time step, the MHE strategy in [10] solves

$$
\mathcal{P}_{t}:\left\{\begin{array}{l}
\widehat{x}_{t-N, t}^{o}=\arg \min J_{t} \\
\text { s.t. } \widehat{x}_{i+1, t}=A \widehat{x}_{i, t}+B u_{i}, i \in \mathscr{I}_{t-N}^{t-1} \\
\quad \widehat{y}_{i, t}=C \widehat{x}_{i, t}, \quad i \in \mathscr{I}_{t-N}^{t}
\end{array},\right.
$$

where,

$$
J_{t}=\mu\left\|\widehat{x}_{t-N, t}-\bar{x}_{t-N, t}\right\|^{2}+\sum_{i=t-N}^{t}\left\|y_{i}-C \widehat{x}_{i, t}\right\|^{2},
$$

with $\mu \geqslant 0, \bar{x}_{t-N}$ is an a priori estimation of $x_{t-N}, N \geq n$. In [10], it is assumed that for $t=N$, one already has $\bar{x}_{0}$ as a priori estimate; for $t=N+1, N+2, \cdots$, the priori estimation $\bar{x}_{t-N}$ is updated via $\bar{x}_{t-N, t}=A \widehat{x}_{t-N-1, t-1}^{o}+B u_{t-N-1}$, where $\widehat{x}_{t-N-1, t-1}^{o}$ is the optimal estimate at the previous estimation step. Motivated by the fact that $\widehat{x}_{i+1, t}$ is obtained by updating the system dynamics from $\widehat{x}_{i, t}$ in open-loop in (17), [11] has proposed to embed a Luenberger observer into (17):

$$
\overline{\mathcal{P}}_{t}:\left\{\begin{array}{l}
\widehat{x}_{t-N, t}^{o}=\arg \min \bar{J}_{t} \\
\text { s.t. } \widehat{x}_{i+1, t}=A \widehat{x}_{i, t}+B u_{i} \\
+L\left(y_{i}-\widehat{y}_{i, t}\right), i \in \mathscr{I}_{t-N}^{t-1} \\
\widehat{y}_{i, t}=C \widehat{x}_{i, t}, \quad i \in \mathscr{I}_{t-N}^{t}
\end{array},\right.
$$

where,

$$
\bar{J}_{t}=\mu\left\|\widehat{x}_{t-N, t}-\bar{x}_{t-N, t}\right\|^{2}+\left\|W\left(\mathbf{y}_{t-N}^{t}-\widehat{\mathbf{y}}_{t-N}^{t, t}\right)\right\|^{2},
$$

with $\mu \geqslant 0, W \in \mathbf{R}^{n \times(N+1) p}$, and

$$
\mathbf{y}_{t-N}^{t}=\left[y_{t-N}, \cdots, y_{t}\right], \widehat{\mathbf{y}}_{t-N}^{t, t}=\left[\widehat{y}_{t-N, t}, \cdots, \widehat{y}_{t, t}\right]
$$

and $L$ is chosen such that $A_{L}=A-L C$ is Schur stable. Different from [10], for $t=N+1, N+2, \cdots$, the priori estimation $\bar{x}_{t-N, t}$ in [11] is updated via

$$
\left\{\begin{array}{l}
\bar{x}_{t-N, t}=A \widehat{x}_{t-N-1, t-1}^{o}+B u_{t-N-1} \\
+L\left(y_{t-N-1}-\widehat{y}_{t-N-1, t-1}^{o}\right) \\
\widehat{y}_{t-N-1, t-1}^{o}=C \widehat{x}_{t-N-1, t-1}^{o}
\end{array},\right.
$$

where $\widehat{x}_{t-N-1, t-1}^{o}$ is the optimal estimate at the previous estimation step and $\bar{x}_{0, N}=\bar{x}_{0}$. [11] has shown that the convergence of the MHE (18) only depends on the Schur stability of $A_{L}$ and is independent of $\mu$, given the introduction of the pre-estimation.

\section{A. A parameterized cost for MHE with pre-estimation}

The MHE problem we consider takes the form of (18), with $\bar{J}_{t}$ being replaced by

$$
\begin{aligned}
& \vec{J}_{t}=(1-\lambda) \bar{\mu}\left\|\widehat{x}_{t-N, t}-\bar{x}_{t-N, t}\right\|^{2} \\
& +\lambda\left(\sum_{i=t-N}^{t}\left\|y_{i}-C \widehat{x}_{i, t}\right\|^{2}+\mu\left\|\widehat{x}_{t-N, t}-\bar{x}_{t-N, t}\right\|^{2}\right),
\end{aligned}
$$

with $\bar{\mu}, \mu \geqslant 0, \lambda \in[0,1]$. We consider the simple case with scaler weightings without constraints. The results can be extended to general cases with matrix weightings and constraints [12]. As in [11], the priori estimation $\bar{x}_{t-N, t}$ is updated via (20). We firstly rewrite $\vec{J}_{t}$

$$
\vec{J}_{t}=\bar{\lambda}\left\|\widehat{x}_{t-N, t}-\bar{x}_{t-N, t}\right\|^{2}+\lambda\left\|\mathbf{y}_{t-N}^{t}-\widehat{\mathbf{y}}_{t-N}^{t, t}\right\|^{2},
$$

where,

$$
\bar{\lambda}=\lambda \mu+(1-\lambda) \bar{\mu} .
$$

When $\lambda=0, \vec{J}_{t}\left(\bar{x}_{t-N, t}, \mathcal{I}_{t}\right)=\bar{\mu}\left\|\widehat{x}_{t-N, t}-\bar{x}_{t-N, t}\right\|^{2}$, and the optimal estimate $\widehat{x}_{t-N, t}^{o}=\bar{x}_{t-N, t}$, i.e., the proposed strategy matches the existing Luenberger observer. When $\lambda=1$, the term $(1-\lambda)\left\|\widehat{x}_{t-N, t}-\bar{x}_{t-N, t}\right\|^{2}$ disappears, i.e., the proposed method reduces to a similar MHE strategy with that of [11]. In general, $\bar{\mu}$ and $\mu$ are two nonnegative scalars to be selected by the user. The special cases of when they are equal, or when one of them is zero with the other being positive, can be analyzed easily. In the following, we consider the case of $\lambda \in(0,1)$ with $\bar{\mu}$ and $\mu$ having different positive values. For this case, the increase of $\lambda$ stands for the designers' desire to forget relatively old data and make sure the nominal model prediction track the new measurements closely. This can be more clearly seen if we divide both sides of (21) by $\lambda$, i.e.,

$$
\begin{aligned}
& \widetilde{J}_{t}=\lambda^{-1} \vec{J}_{t} \\
& =\lambda^{-1} \bar{\lambda}\left\|\widehat{x}_{t-N, t}-\bar{x}_{t-N, t}\right\|^{2}+\left\|\mathbf{y}_{t-N}^{t}-\widehat{\mathbf{y}}_{t-N}^{t, t}\right\|^{2} .
\end{aligned}
$$

In $\widetilde{J}_{t}$, the weightings on $\left\|\widehat{x}_{t-N, t}-\bar{x}_{t-N, t}\right\|^{2}$ and $\left\|\mathbf{y}_{t-N}^{t}-\widehat{\mathbf{y}}_{t-N}^{t, t}\right\|^{2}$ is $\lambda^{-1} \bar{\lambda}$ and 1 , respectively.

Proposition 2: Assume $\lambda \in(0,1), \bar{\mu}$ and $\mu$ take different positive values. Then the following results hold:

(i) if $(1-\lambda) \bar{\mu}=\lambda(1-\mu)$, then $\lambda^{-1} \bar{\lambda}=1$; a necessary condition for $\lambda^{-1} \bar{\lambda}=1$ is that $0<\mu<1$;

(ii) if $(1-\lambda) \bar{\mu}>\lambda(1-\mu)$, then $\lambda^{-1} \bar{\lambda}>1$; moreover, if $\mu \geq 1$, one always has $\lambda^{-1} \bar{\lambda}>1$;

(iii) if $(1-\lambda) \bar{\mu}<\lambda(1-\mu)$, then $\lambda^{-1} \bar{\lambda}<1$; a necessary condition for $\lambda^{-1} \bar{\lambda}<1$ is that $0<\mu<1$;

(iv) one always has $\frac{d\left(\lambda^{-1} \bar{\lambda}\right)}{d \lambda}<0$.

Proof. (i): Assume that $\lambda \in(0,1), \bar{\mu}$ and $\mu$ take different positive values. $\lambda^{-1} \bar{\lambda}=1$ is equivalent to $\lambda \mu+(1-\lambda) \bar{\mu}=\lambda$, which reduces to $(1-\lambda) \bar{\mu}=\lambda(1-\mu)$. Since $(1-\lambda) \bar{\mu}>0$ for $\lambda \in(0,1), \bar{\mu}>0$, one must have that $\lambda(1-\mu)>0$. 
Therefore, it must hold that $0<\mu<1$. Part (i) is proved. (ii) and (iii): These results can be proved by following similar arguments with part (i). (iv): It can be derived

$$
\frac{d\left(\lambda^{-1} \bar{\lambda}\right)}{d \lambda}=-\frac{\bar{\mu}}{\lambda^{2}}<0,
$$

for $\lambda \in(0,1), \bar{\mu}>0$. This completes the proof.

In the following, we consider the MHE problem (18) with cost $\vec{J}_{t}$ being replaced by $\widetilde{J}_{t}(22)$. Doing so will not affect the optimal solution or stability, since the cost is only changed by a positive scalar. Denote

$$
\mathbf{C}=\operatorname{diag}_{N+1}(C, \cdots, C)
$$

and $\widehat{x}_{t-N, t}^{o}$ as the optimal solution to the MHE problem 18 with cost $\widetilde{J}_{t}(22)$, and the estimation error as

$$
e_{t-N}=x_{t-N}-\widehat{x}_{t-N, t}^{o} .
$$

\section{B. Estimation error analysis}

Denote

$$
\begin{aligned}
& \widehat{\mathbf{x}}_{t-N}^{t, t}=\left[\widehat{x}_{t-N, t}, \cdots, \widehat{x}_{t, t}\right], \mathbf{x}_{t-N}^{t}=\left[x_{t-N}, \cdots, x_{t}\right], \\
& \mathbf{w}_{t-N}^{t-1}=\left[w_{t-N}, \cdots, w_{t-1}\right], \mathbf{v}_{t-N}^{t}=\left[v_{t-N}, \cdots, v_{t}\right], \\
& \mathbf{u}_{t-N}^{t-1}=\left[u_{t-N}, \cdots, u_{t-1}\right] .
\end{aligned}
$$

From (16), one can obtain

$$
\mathbf{y}_{t-N}^{t}=\boldsymbol{\Lambda} x_{t-N}+\boldsymbol{\Gamma} \mathbf{u}_{t-N}^{t-1}+\boldsymbol{\Phi} \mathbf{w}_{t-N}^{t-1}+\mathbf{v}_{t-N}^{t},
$$

where, $\mathbf{y}_{t-N}^{t}, \mathbf{x}_{t-N}^{t}, \mathbf{u}_{t-N}^{t-1}, \mathbf{w}_{t-N}^{t-1}, \mathbf{v}_{t-N}^{t}$ are defined in 19 ) and before (23), respectively, and $\boldsymbol{\Lambda}, \boldsymbol{\Gamma}, \boldsymbol{\Phi}$ can be found in [11]. Rewriting the Luenberger observer's dynamics in (18) gives

$$
\widehat{x}_{i+1, t}=A_{L} \widehat{x}_{i, t}+B u_{i}+L y_{i} .
$$

Then, it can be verified that

$$
\widehat{\mathbf{y}}_{t-N}^{t, t}=\mathbf{C} \widehat{\mathbf{x}}_{t-N}^{t, t}=\overline{\mathbf{\Lambda}} \widehat{x}_{t-N, t}+\overline{\mathbf{\Gamma}} \mathbf{u}_{t-N}^{t-1}+\overline{\mathbf{\Phi}} \mathbf{y}_{t-N}^{t},
$$

where, $\widehat{\mathbf{y}}_{t-N}^{t, t}, \mathbf{y}_{t-N}^{t}, \widehat{\mathbf{x}}_{t-N}^{t, t}, \mathbf{u}_{t-N}^{t-1}$ are defined in (19) and before (23), respectively, and $\bar{\Lambda}, \bar{\Gamma}, \bar{\Phi}$ can be found in [11]. Denote $\Psi=I_{(N+1) p}-\mathbf{L}_{N}$ with

$$
\mathbf{L}_{N}=\left[\begin{array}{ccccc}
0 & 0 & \cdots & 0 & 0 \\
C L & 0 & \cdots & 0 & 0 \\
C A_{L} L & C L & \cdots & 0 & 0 \\
\vdots & \vdots & \ddots & \vdots & \vdots \\
C A_{L}^{N-1} L & C A_{L}^{N-2} L & \cdots & C L & 0
\end{array}\right] .
$$

We then have the following results on the error dynamics.

Proposition 3: Assume that $\lambda \in(0,1), \bar{\mu}$ and $\mu$ take different positive values. Then the estimation error dynamics takes the following form

$$
e_{t-N}=\bar{A}_{L} e_{t-N-1}+S^{-1} S_{1} \mathbf{w}_{t-N-1}^{t-1}+S^{-1} S_{2} \mathbf{v}_{t-N-1}^{t},
$$

where,

$$
\begin{aligned}
& \bar{A}_{L}=\lambda^{-1} \overline{\lambda S}^{-1} A_{L}, \bar{S}=\lambda^{-1} \bar{\lambda} I+\overline{\boldsymbol{\Lambda}}^{\mathrm{T}} \overline{\boldsymbol{\Lambda}} \\
& S_{1}=\left[\begin{array}{ll}
\lambda^{-1} \bar{\lambda} I & -\overline{\boldsymbol{\Lambda}}^{\mathrm{T}} \overline{\boldsymbol{\Phi}}
\end{array}\right], S_{2}=\left[\begin{array}{ll}
-\lambda^{-1} \bar{\lambda} L & -\overline{\boldsymbol{\Lambda}}^{\mathrm{T}} \Psi
\end{array}\right] \\
& \mathbf{w}_{t-N-1}^{t-1}=\left[w_{t-N-1}, \mathbf{w}_{t-N}^{t-1}\right], \mathbf{v}_{t-N-1}^{t}=\left[v_{t-N-1}, \mathbf{v}_{t-N}^{t}\right] \text {. }
\end{aligned}
$$

Proof. The proof follows a similar procedure with that of Theorem 1 of [11] and is skipped here.

Theorem 2: Assume $A_{L}$ is Schur stable, $\lambda \in(0,1), \bar{\mu}$ and $\mu$ take different positive values. Then we have (i) $\bar{A}_{L}$ is Schur stable; (ii) without process and measurement noises, the estimation error exponentially converges to zero; (iii) without process and measurement noises, when either of the following two conditions is satisfied, $0<\mu<\bar{\mu}, \bar{\mu}<\mu \leq \bar{\mu}+\frac{1}{\lambda}$, the decaying rate of the estimation error is monotonically increasing w.r.t. $\lambda$.

Proof. (i): If $A_{L}$ is Schur stable, for $Q_{L}>0$, there exists a unique solution $P_{L}>0$ satisfying the following Lyapunov equation

$$
A_{L}^{\mathrm{T}} P_{L} A_{L}-P_{L}+Q_{L}=0 .
$$

From Proposition 3, one has

$$
\bar{A}_{L}=\lambda^{-1} \overline{\lambda S}^{-1} A_{L}=\left(I+\tilde{\lambda} \overline{\boldsymbol{\Lambda}}^{\mathrm{T}} \overline{\boldsymbol{\Lambda}}\right)^{-1} A_{L},
$$

with

$$
\widetilde{\lambda}=\frac{\lambda}{\bar{\lambda}}
$$

With the above, (25) can be rewritten as

$$
\bar{A}_{L}^{\mathrm{T}}\left(I+\tilde{\lambda} \overline{\boldsymbol{\Lambda}}^{\mathrm{T}} \overline{\boldsymbol{\Lambda}}\right) P_{L}\left(I+\tilde{\lambda} \overline{\boldsymbol{\Lambda}}^{\mathrm{T}} \overline{\boldsymbol{\Lambda}}\right) \bar{A}_{L}-\bar{P}_{L}+Q_{L}=0,
$$

i.e., $\bar{A}_{L}^{\mathrm{T}} \bar{P}_{L} \bar{A}_{L}-\bar{P}_{L}+\bar{Q}_{L}=0$, with

$$
\begin{aligned}
& \bar{P}_{L}=\left(I+\widetilde{\lambda} \overline{\boldsymbol{\Lambda}}^{\mathrm{T}} \overline{\boldsymbol{\Lambda}}\right) P_{L}\left(I+\widetilde{\lambda} \overline{\boldsymbol{\Lambda}}^{\mathrm{T}} \overline{\boldsymbol{\Lambda}}\right)>0, \\
& \bar{Q}_{L}=\left(I+\widetilde{\lambda} \overline{\boldsymbol{\Lambda}}^{\mathrm{T}} \overline{\boldsymbol{\Lambda}}\right) P_{L}\left(I+\widetilde{\lambda} \overline{\boldsymbol{\Lambda}}^{\mathrm{T}} \overline{\boldsymbol{\Lambda}}\right)-P_{L}+Q_{L} .
\end{aligned}
$$

Thus, part (i) can be established if $\bar{Q}_{L}>0$. Given $Q_{L}>0$, the positive definiteness of $\bar{Q}_{L}$ depends on $\left(I+\widetilde{\lambda} \overline{\boldsymbol{\Lambda}}^{\mathrm{T}} \overline{\boldsymbol{\Lambda}}\right) P_{L}(I+$ $\left.\widetilde{\lambda} \overline{\boldsymbol{\Lambda}}^{\mathrm{T}} \overline{\boldsymbol{\Lambda}}\right)-P_{L}$. Since $(A, C)$ is observable and $N \geq n$, one has $\overline{\boldsymbol{\Lambda}}^{\mathrm{T}} \overline{\boldsymbol{\Lambda}}>0$. Therefore, we have

$$
\bar{Q}_{L}=\widetilde{\lambda}\left(P_{L} \overline{\boldsymbol{\Lambda}}^{\mathrm{T}} \overline{\boldsymbol{\Lambda}}+\overline{\boldsymbol{\Lambda}}^{\mathrm{T}} \overline{\boldsymbol{\Lambda}} P_{L}\right)+\widetilde{\lambda}^{2} \overline{\boldsymbol{\Lambda}}^{\mathrm{T}} \overline{\boldsymbol{\Lambda}} P_{L} \overline{\boldsymbol{\Lambda}}^{\mathrm{T}} \overline{\boldsymbol{\Lambda}}+Q_{L} .
$$

Note that $\widetilde{\lambda}^{2} \overline{\boldsymbol{\Lambda}}^{\mathrm{T}} \overline{\boldsymbol{\Lambda}} P_{L} \overline{\boldsymbol{\Lambda}}^{\mathrm{T}} \overline{\boldsymbol{\Lambda}}>0$. We will just have to prove that $P_{L} \overline{\boldsymbol{\Lambda}}^{\mathrm{T}} \overline{\boldsymbol{\Lambda}}+\overline{\boldsymbol{\Lambda}}^{\mathrm{T}} \overline{\boldsymbol{\Lambda}} P_{L}>0$. To do so, we show all the eigenvalues of $P_{L} \bar{\Lambda}^{\mathrm{T}} \overline{\boldsymbol{\Lambda}}$ are positive. Assume $\gamma$ is an eigenvalue of $P_{L} \overline{\boldsymbol{\Lambda}}^{\mathrm{T}} \overline{\boldsymbol{\Lambda}}$ with the associated eigenvector $\tau \neq 0$, i.e., $P_{L} \overline{\boldsymbol{\Lambda}}^{\mathrm{T}} \overline{\boldsymbol{\Lambda}} \tau=\gamma \tau$. Multiplying both sides of the above equation from the left by $\overline{\boldsymbol{\Lambda}}^{\mathrm{T}} \overline{\boldsymbol{\Lambda}}$ gives us $\overline{\boldsymbol{\Lambda}}^{\mathrm{T}} \overline{\boldsymbol{\Lambda}} P_{L} \overline{\boldsymbol{\Lambda}}^{\mathrm{T}} \overline{\boldsymbol{\Lambda}} \tau=\gamma \overline{\boldsymbol{\Lambda}}^{\mathrm{T}} \overline{\boldsymbol{\Lambda}} \tau$. This further implies that $\tau^{\mathrm{T}} \overline{\boldsymbol{\Lambda}}^{\mathrm{T}} \overline{\boldsymbol{\Lambda}} P_{L} \overline{\boldsymbol{\Lambda}}^{\mathrm{T}} \overline{\boldsymbol{\Lambda}} \tau=\gamma \tau^{\mathrm{T}} \overline{\boldsymbol{\Lambda}}^{\mathrm{T}} \overline{\boldsymbol{\Lambda}} \tau$, i.e.,

$$
\gamma=\frac{\tau^{\mathrm{T}} \overline{\boldsymbol{\Lambda}}^{\mathrm{T}} \overline{\boldsymbol{\Lambda}} P_{L} \overline{\boldsymbol{\Lambda}}^{\mathrm{T}} \overline{\boldsymbol{\Lambda}} \tau}{\tau^{\mathrm{T}} \overline{\boldsymbol{\Lambda}}^{\mathrm{T}} \overline{\boldsymbol{\Lambda}} \tau}>0
$$

given $\overline{\boldsymbol{\Lambda}}^{\mathrm{T}} \overline{\boldsymbol{\Lambda}} P_{L} \overline{\boldsymbol{\Lambda}}^{\mathrm{T}} \overline{\boldsymbol{\Lambda}}>0$ and $\overline{\boldsymbol{\Lambda}}^{\mathrm{T}} \overline{\boldsymbol{\Lambda}}>0$. Thus, all the eigenvalues of $P_{L} \overline{\boldsymbol{\Lambda}}^{\mathrm{T}} \overline{\boldsymbol{\Lambda}}$ are positive, although $P_{L} \overline{\boldsymbol{\Lambda}}^{\mathrm{T}} \overline{\boldsymbol{\Lambda}}$ is not symmetric in general. Similarly, all the eigenvalues of $\bar{\Lambda}^{\mathrm{T}} \bar{\Lambda} P_{L}$ are positive, i.e., $\widetilde{\lambda}\left(P_{L} \overline{\boldsymbol{\Lambda}}^{\mathrm{T}} \overline{\boldsymbol{\Lambda}}+\overline{\boldsymbol{\Lambda}}^{\mathrm{T}} \overline{\boldsymbol{\Lambda}} P_{L}\right)>0$. Thus, one has $\bar{Q}_{L}>0$ and $\bar{A}_{L}$ is Schur stable. (ii) Without process and measurement noises, the estimation error dynamics reduces to

$$
e_{t+1}=\bar{A}_{L} e_{t}
$$

for $t=0,1,2,3, \cdots$. Based on part (i), the estimation error exponentially converges to zero. (iii) Denote

$$
V_{e_{t}}=e_{t}^{\mathrm{T}} \bar{P}_{L} e_{t}
$$


as the Lyapunov function for (27). It holds that

$$
\Delta V_{e_{t}}=V_{e_{t+1}}-V_{e_{t}}=-e_{t}^{\mathrm{T}} \bar{Q}_{L} e_{t} .
$$

Therefore, part (iii) will be proved if $\frac{d \bar{Q}_{L}}{d \lambda}>0$. For $\bar{Q}_{L}$, we have

$$
\frac{d \bar{Q}_{L}}{d \lambda}=\left(P_{L} \overline{\boldsymbol{\Lambda}}^{\mathrm{T}} \overline{\boldsymbol{\Lambda}}+\overline{\boldsymbol{\Lambda}}^{\mathrm{T}} \overline{\boldsymbol{\Lambda}} P_{L}\right) \frac{d \tilde{\lambda}}{d \lambda}+\overline{\boldsymbol{\Lambda}}^{\mathrm{T}} \overline{\boldsymbol{\Lambda}} P_{L} \overline{\boldsymbol{\Lambda}}^{\mathrm{T}} \overline{\boldsymbol{\Lambda}} \frac{d \widetilde{\lambda}^{2}}{d \lambda} .
$$

It can be verified that

$$
\frac{d \widetilde{\lambda}}{d \lambda}=\frac{\bar{\mu}}{\bar{\lambda}^{2}}, \frac{d\left(\widetilde{\lambda}^{2}\right)}{d \lambda}=\frac{2 \lambda(1-\lambda(\mu-\bar{\mu}))}{\bar{\lambda}^{3}} .
$$

Obviously, one has $\frac{d \widetilde{\lambda}}{d \lambda}>0$. Therefore, if $1-\lambda(\mu-\bar{\mu}) \geq 0$, one always has $\frac{d \bar{Q}_{L}}{d \lambda}>0$. Given $\lambda \in(0,1)$ and $\mu \neq \bar{\mu}$, there are two conditions under which the above inequality holds, namely, $0<\mu<\bar{\mu}$, or $\bar{\mu}<\mu \leq \bar{\mu}+\frac{1}{\lambda}$.

Given $\bar{A}_{L}=\lambda^{-1} \overline{\lambda S}^{-1} A_{L}$ and $\bar{S}>0$, even it has been proved in Proposition 2 that $\frac{d\left(\lambda^{-1} \bar{\lambda}\right)}{d \lambda}<0$, it is not necessarily true that the magnitude of the eigenvalues of $\bar{A}_{L}$ will decrease with one increases $\lambda$. This can be more clearly seen as follows. Note that we can rewrite

$$
\bar{A}_{L}=\left(1+\left(\lambda^{-1} \bar{\lambda}\right)^{-1} \overline{\boldsymbol{\Lambda}}^{\mathrm{T}} \overline{\boldsymbol{\Lambda}}\right)^{-1} A_{L}=\widetilde{\boldsymbol{\Lambda}}^{-1} A_{L} .
$$

Therefore, it holds that

$$
\begin{aligned}
& \frac{d \bar{A}_{L}}{d \lambda}=-\widetilde{\boldsymbol{\Lambda}}^{-1} \frac{d \widetilde{\mathbf{\Lambda}}}{\frac{d}{\lambda}} \widetilde{\boldsymbol{\Lambda}}^{-1} A_{L} \\
& =-\widetilde{\boldsymbol{\Lambda}}^{-1} \frac{d\left(\lambda^{-1} \frac{d \lambda}{\lambda}\right)^{-1}}{d \lambda} \overline{\boldsymbol{\Lambda}}^{\mathrm{T}} \overline{\boldsymbol{\Lambda}} \widetilde{\boldsymbol{\Lambda}}^{-1} A_{L} \\
& =\overrightarrow{\boldsymbol{\Lambda}} A_{L},
\end{aligned}
$$

where, $\overrightarrow{\boldsymbol{\Lambda}}<0$, since $\frac{d\left(\lambda^{-1} \bar{\lambda}\right)}{d \lambda} \leq 0$. However, whether the magnitudes of the eigenvalues of $\bar{A}_{L}$ increase or decrease can not be verified. What we can prove is that

$$
\frac{d \Delta V_{e_{t}}}{d \lambda}=-e_{t}^{\mathrm{T}} \frac{d \bar{Q}_{L}}{d \lambda} e_{t}<0,
$$

since one always has $\frac{d \bar{Q}_{L}}{d \lambda}>0$, e.g., the decaying rate of the error dynamics without disturbances can be increased monotonically by increasing $\lambda$ with proper selections of $\mu$ and $\bar{\mu}$.

In the following, we consider the case with bounded disturbances. Denote

$$
z_{w}=\max _{w_{t} \in \mathcal{W}}\left\|w_{t}\right\|, z_{v}=\max _{v_{t} \in \mathcal{V}}\left\|v_{t}\right\| .
$$

Given $(A, C)$ is observable in $N$ steps, one has $\overline{\boldsymbol{\Lambda}}^{\mathrm{T}} \overline{\boldsymbol{\Lambda}}>0$ with $\bar{\Lambda}$ being defined in (24). Although the eigenvalues of $\bar{\Lambda}^{\mathrm{T}} \bar{\Lambda}$ is dependent on the choice of $L$, without loss of generality, it is reasonable for us to assume that

$$
\overline{\boldsymbol{\Lambda}}^{\mathrm{T}} \overline{\boldsymbol{\Lambda}} \geq \eta I
$$

where $\eta$ is a certain positive number.

Theorem 3: Assume $A_{L}$ is Schur stable, $\lambda \in(0,1), \bar{\mu}$ and $\mu$ take different positive values. We have:

(i) for $t=N, N+1, \cdots$, the estimation error is bounded by $\left\|e_{t-N}\right\| \leq \zeta_{t-N}$, where, $\zeta_{t-N}$ is a sequence generated by

$$
\zeta_{t}=a \zeta_{t-1}+b, \zeta_{0}=b_{0},
$$

in which,

$$
\begin{aligned}
& a=a_{l}\left\|\lambda^{-1} \overline{\lambda S}^{-1}\right\|, \quad b=\left\|\lambda^{-1} \overline{\lambda S}^{-1}\right\| \bar{z}+\left\|\bar{S}^{-1}\right\| \bar{\theta}, \\
& b_{0}=\left\|I-\bar{S}^{-1} \overline{\boldsymbol{\Lambda}}^{\mathrm{T}} \overline{\boldsymbol{\Lambda}}\right\|\left\|x_{0}\right\|+\left\|\lambda^{-1} \overline{\lambda S} \bar{S}^{-1}\right\|\left\|\bar{x}_{0}\right\|+\left\|\bar{S}^{-1}\right\| \bar{\theta},
\end{aligned}
$$

with

$$
\begin{aligned}
& \bar{z}=z_{w}+l z_{v}, \bar{\theta}=\theta_{1} \sqrt{N} z_{w}+\theta_{2} \sqrt{N+1} z_{v} \\
& a_{l}=\left\|A_{L}\right\|, \theta_{1}=\left\|\overline{\boldsymbol{\Lambda}}^{\mathrm{T}} \overline{\mathbf{\Phi}}\right\|, l=\|L\|, \theta_{2}=\left\|\overline{\boldsymbol{\Lambda}}^{\mathrm{T}} \Psi\right\| .
\end{aligned}
$$

(ii) if

$$
\frac{a_{l} \sqrt{n}}{1+\widetilde{\lambda} \eta}<1,
$$

with $n$ as the dimension of the state, and $\widetilde{\lambda}, \eta, a_{l}$ being defined in (26), (29), (30), respectively, then $a<1$ and the sequence $\left\{\zeta_{t}\right\}$ converges exponentially to

$$
\zeta_{\infty}=\frac{b}{1-a} .
$$

Proof. (i) This part can be proved by using the error dynamics given in Proposition 3 , following similar steps as those in Theorem 3 of [11], and recognizing that $\|A+B\| \leq\|A\|+\|B\|$, $\|A B\| \leq\|A\|\|B\|$, where, $A$ and $B$ are vectors or matrices of proper dimensions. (ii) The convergence of sequence $\left\{\zeta_{t}\right\}$ (30) depends on $a<1$. When $a<1, \zeta_{\infty}=\frac{b}{1-a}$, as $t \rightarrow \infty$. Note that

$$
\begin{aligned}
& a=a_{l}\left\|\lambda^{-1} \bar{\lambda} S^{-1}\right\|=a_{l} \sqrt{\operatorname{tr}\left(\lambda^{-1} \bar{\lambda} S^{-1} \lambda^{-1} \bar{\lambda} S^{-1}\right)} \\
& =a_{l} \sqrt{\operatorname{tr}\left(\left(I+\widetilde{\lambda} \overline{\boldsymbol{\Lambda}}^{\mathrm{T}} \overline{\boldsymbol{\Lambda}}\right)^{-1}\left(I+\widetilde{\lambda} \overline{\boldsymbol{\Lambda}}^{\mathrm{T}} \overline{\boldsymbol{\Lambda}}\right)^{-1}\right)},
\end{aligned}
$$

with $\widetilde{\lambda}$ being defined in (26). Note that

$$
\begin{aligned}
& \lambda_{\min }\left(M_{1}\right) \lambda_{\max }\left(M_{2}\right) \leq \lambda_{\min }\left(M_{1}\right) \operatorname{tr}\left(M_{2}\right) \\
& \leq \operatorname{tr}\left(M_{1} M_{2}\right) \leq \lambda_{\max }\left(M_{1}\right) \operatorname{tr}\left(M_{2}\right),
\end{aligned}
$$

for two positive definite matrices $M_{1}$ and $M_{2}$ [21]. Since $\overline{\boldsymbol{\Lambda}}^{\mathrm{T}} \overline{\boldsymbol{\Lambda}} \geq \eta I$, one has that

$$
I+\widetilde{\lambda} \overline{\boldsymbol{\Lambda}}^{\mathrm{T}} \overline{\boldsymbol{\Lambda}} \geq(1+\tilde{\lambda} \eta) I,
$$

i.e.,

$$
\operatorname{tr}\left(\left(I+\widetilde{\lambda} \overline{\boldsymbol{\Lambda}}^{\mathrm{T}} \overline{\boldsymbol{\Lambda}}\right)^{-1}\right) \leq n \lambda_{\max }\left(\left(I+\widetilde{\lambda} \overline{\boldsymbol{\Lambda}}^{\mathrm{T}} \overline{\boldsymbol{\Lambda}}\right)^{-1}\right)=\frac{n}{1+\widetilde{\lambda} \eta} .
$$

It can be further derived that

$$
a \leq a_{l} \sqrt{\lambda_{\max }\left(\left(I+\widetilde{\lambda} \overline{\boldsymbol{\Lambda}}^{\mathrm{T}} \overline{\boldsymbol{\Lambda}}\right)^{-1}\right) \frac{n}{1+\widetilde{\lambda} \eta}} \leq \frac{a_{l} \sqrt{n}}{1+\widetilde{\lambda} \eta} .
$$

Therefore, if (31) is satisfied, we will have $a<1$.

An important question here is whether $\lambda$ can be tuned to possibly reduce $\zeta_{\infty}$. This motivates us to derive

$$
\frac{d \zeta_{\infty}}{d \lambda}=\frac{1}{(1-a)^{2}}\left[(1-a) \frac{d b}{d \lambda}+b \frac{d a}{d \lambda}\right] .
$$

It is not obvious to ascertain whether $\frac{d \zeta_{\infty}}{d \lambda}$ is positive or negative when $\lambda \in(0,1)$, because $a$ and $b$ are parameterized by $\lambda$ in a complicated way. To circumvent this difficulty, we set our sights lower and define a normalized sequence by dividing both sides of (30) with $b: \bar{\zeta}_{t}=a \bar{\zeta}_{t-1}+1, \bar{\zeta}_{0}=\frac{b_{0}}{b}$, in which,

$$
\bar{\zeta}_{t}=\frac{\zeta_{t}}{b}, \bar{\zeta}_{t-1}=\frac{\zeta_{t-1}}{b}
$$


If we define $\bar{e}_{t-N}=\frac{e_{t-N}}{b}$, it holds that $\left\|\bar{e}_{t-N}\right\| \leq \bar{\zeta}_{t-N}$. We then have the following results.

Theorem 4: Assume $A_{L}$ is Schur stable, $\lambda \in(0,1), \bar{\mu}$ and $\mu$ take different positive values. If (31) is satisfied, the normalized bounding sequence $\left\{\bar{\zeta}_{t}\right\}$ converges exponentially to the following value $\bar{\zeta}_{\infty}=\frac{1}{1-a}$. Moreover, $\bar{\zeta}_{\infty}$ decreases monotonically when one increases $\lambda$.

Proof. The convergence of $\left\{\bar{\zeta}_{t}\right\}$ to $\bar{\zeta}_{\infty}$ can be proved similarly to that of Theorem 3. Note that for a given $M>0$, if $\gamma>0$ is an eigenvalue of $M$ with the associated eigenvector $\tau \neq 0$, i.e., $M \tau=\gamma \tau$, then $\gamma^{2}$ is an eigenvalue of $M^{2}$ with the associated eigenvector $\tau \neq 0$, and $\gamma^{-1}$ is an eigenvalue of $M^{-1}$ with the associated eigenvector $\tau \neq 0$. These can be easily verified as follows

$$
M^{2} \tau=\gamma M \tau=\gamma^{2} \tau, M^{-1} \tau=\gamma^{-1} \tau
$$

Denote $\left\{\gamma_{1}, \gamma_{2}, \cdots, \gamma_{n-1}, \gamma_{n}\right\}$ as the eigenvalues of the matrix $\bar{\lambda} S^{-1}=\left(I+\widetilde{\lambda} \bar{\Lambda}^{\mathrm{T}} \overline{\boldsymbol{\Lambda}}\right)^{-1}$. Then we know that

$$
\operatorname{tr}\left(\left(I+\widetilde{\lambda} \overline{\boldsymbol{\Lambda}}^{\mathrm{T}} \overline{\boldsymbol{\Lambda}}\right)^{-2}\right)=\gamma_{1}^{2}+\gamma_{2}^{2}+\cdots+\gamma_{n-1}^{2}+\gamma_{n}^{2}
$$

Note from (28), we have

$$
\frac{d \widetilde{\lambda}}{d \lambda}=\frac{\bar{\mu}}{\bar{\lambda}^{2}}>0 .
$$

Therefore, eigenvalues of matrix $I+\widetilde{\lambda} \overline{\boldsymbol{\Lambda}}^{\mathrm{T}} \overline{\boldsymbol{\Lambda}}$ increase monotonically when one increases $\lambda$. In other words, eigenvalues of matrix $\left(I+\tilde{\lambda} \overline{\boldsymbol{\Lambda}}^{\mathrm{T}} \overline{\boldsymbol{\Lambda}}\right)^{-1}$ and $\left(I+\tilde{\lambda} \overline{\boldsymbol{\Lambda}}^{\mathrm{T}} \overline{\boldsymbol{\Lambda}}\right)^{-2}$ decrease monotonically when $\lambda$ is increased, i.e,

$$
\begin{aligned}
& \frac{d a}{d \lambda}=a_{l} \frac{d\left\|\lambda^{-1} \bar{\lambda} S^{-1}\right\|}{d \bar{\lambda}} \\
& =a_{l} \frac{d\left\|\left(I+\widetilde{\lambda} \overline{\boldsymbol{\Lambda}}^{\mathrm{T}} \overline{\boldsymbol{\Lambda}}\right)^{-1}\right\|}{d \lambda} \\
& =a_{l} \frac{d \sqrt{\operatorname{tr}\left(\left(I+\widetilde{\lambda} \overline{\boldsymbol{\Lambda}}^{\mathrm{T}} \overline{\boldsymbol{\Lambda}}\right)^{-2}\right)}}{d \lambda}<0 .
\end{aligned}
$$

The fact that $\bar{\zeta}_{\infty}$ is a monotonically decreasing function of $\lambda$ can be obtained by recognizing

$$
\frac{d \bar{\zeta}_{\infty}}{d \lambda}=\frac{1}{(1-a)^{2}} \frac{d a}{d \lambda}<0
$$

\section{ILLUSTRATIVE EXAMPLE}

We illustrate the results with the land-based vehicle example in [3], where detailed information about the system model can be found. In the above model, the first two components of the states are the northerly and easterly positions and the last two are the northerly and easterly velocities, respectively. Different from [3], the heading of the vehicle is assumed to be unknown. Assume the input is zero and the sampling period $T=0.5 \mathrm{~s}$. We design a pre-estimator with

$$
L=\left[\begin{array}{ccc}
1.2466 & 0 & 0 \\
0 & 0.8627 & 0.4358 \\
0.6759 & 0 & 0 \\
0 & 0.0090 & 0.8535
\end{array}\right]
$$

so that the set of eigenvalues of $A_{L}=A-L C$ is $\{0.1519,0.6015,0.1419 \pm 0.0236 i\}$.
Firstly, for system (6), we compare the metamorphic MHE in Section II (with $\lambda$ taking the value of $0.1,0.5$ ) with the the unbiased FIR filter in [13]. As such, we choose $Q=I_{4}$; $R=0.5 I_{3} ; M=10 I_{7}$, and the rolling horizon length to be 20 in both metamorphic MHE and FIR. For metamorphic MHE, the weightings on the initial state estimation error within the rolling horizon is taken to be the steady-state solution to the ARE (9), with $\lambda$ taking different values of 0.1 and 0.5 , respectively. Assume that elements of $w_{k}$ and $\nu_{k}$ are uniformly distributed numbers between $[-0.1,0.1]$ and $[-0.25,0.25]$, respectively. The initial state guess of the system (6) is a realization of zero mean, normally distributed random variable with unit covariance while the true value is $\left[5 \cdot \mathbf{1}_{4}, 10 \cdot \mathbf{1}_{4}\right]$. The evolutions of the northerly position estimation error for both MHE and FIR are illustrated in Figure 1 It can be seen that (i) the metamorphic MHE strategy with $\lambda=0.5$ renders better performance than the strategy with $\lambda=0.1$ in the first few sampling instants; afterwards, their performance are nearly distinguishable (because of the effect of the pre-estimator); (ii) the performance of FIR is roughly comparable with that of MHE, and the FIR seems to give slightly worse performance than MHE after the 25-th sampling instant. In Figure 1 before the 21-st sampling instant, both metamorphic MHE and FIR work as batch least-squares estimators with MHE using and FIR not using the initial guess, respectively.

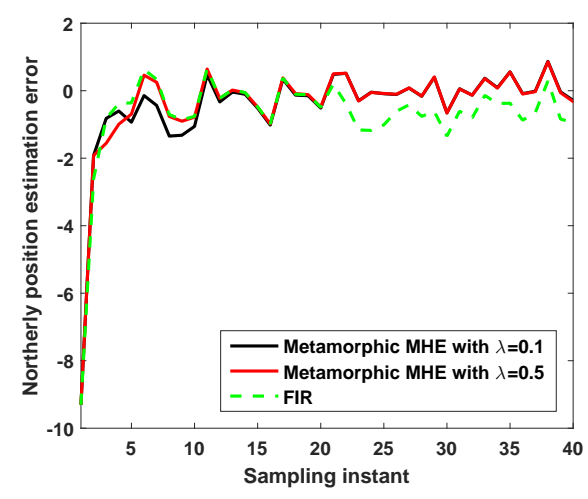

Fig. 1. The evolutions of estimation error

Secondly, using the same example, we compare the MHE strategy in Section III with the the unbiased FIR filter in [13]. For the trajectories of the estimation errors, we notice similar patterns with those shown in Figure 1. Thus, further illustrations are not presented here. Instead, we conduct some numerical analysis of the estimation errors for both strategies. As such, we select $\mu=0.15$ and $\bar{\mu}=0.1$ so that the condition in Theorem 2 is maintained for all $\lambda \in(0,1)$. For MHE, assume that both $x_{0}$ (the true state) and $\bar{x}_{0}$ (the initial guess) are a realization of zero mean, normally distributed random variable with unit covariance. We consider two cases, where elements of the noise vectors are bounded by: (1) $[-0.01,0.01],(2)[-0.025,0.025]$, respectively. We take the initial step as the 0 -th time instant. Starting from the 20-th time instant, we calculate the estimation error for 100 steps when $\lambda$ takes the value of $0,0.25,0.5$, and 0.75 , respectively. When 
TABLE I

ARMSE COMPARISON OF DIFFERENT SCENARIOS $(\mu=0.15, \bar{\mu}=0.1)$

\begin{tabular}{cccccc}
\hline case & $\lambda=0$ & $\lambda=0.25$ & $\lambda=0.5$ & $\lambda=0.75$ & FIR \\
1 & 0.388 & 0.162 & 0.113 & 0.091 & 0.121 \\
2 & 0.3901 & 0.163 & 0.116 & 0.095 & 0.151
\end{tabular}

$\mu=0.15$ and $\bar{\mu}=0.1$, it can be verified that from Proposition 2 that $(1-\lambda) \bar{\mu}<\lambda(1-\mu)$ for $0.25,0.5,0.75$, therefore, the MHE has a forgetting towards the pre-estimate. We repeat the above simulation for 1000 random scenarios. The average root mean square errors (ARMSE) for the MHE and FIR strategies, are shown in Table \. From Table \, we can conclude: (i) with the increase of $\lambda$, MHE gradually improves performance; (ii) the unbiased FIR filter performs roughly as well as MHE with $\lambda \in(0.25,0.5)$, although theoretical comparisons between the two are hard to obtain.

\section{CONCLUSION}

We have proposed a MHE methodology with pre-estimation and normalized forgetting/discounting effects. This is achieved by the introduction of a cost formulation parameterized by a design parameter $\lambda \in[0,1]$. We have examined the idea in two general MHE frameworks. When $\lambda=0$, the proposed technique reduces to the existing estimator. When $\lambda$ is increased, the technique has a more aggressive forgetting effect towards the old data, in a normalized sense. Therefore, when one increases $\lambda$, the proposed framework gradually improves the estimation performance, based on the pre-estimator. Extension of the method to the nonlinear case poses no conceptual difficulty, although establishment of theoretical results would be more involved.

\section{ACKNOWLEDGMENT}

The authors thank the reviewers and Editors for their constructive comments which helped to improve this paper's quality. The first author is grateful to Prof. Graham Goodwin and A/Prof Maria Seron at University of Newcastle, Australia, for sharing illuminating thoughts on constrained estimation. He would also like to thank Dr. Shunyi Zhao at Jiangnan University, China, for helpful discussions on finite impulse response filtering.

\section{REFERENCES}

[1] G. Dissanayake, S. Sukkarieh, E. Nebot, and H. Durrant-Whyte, The aiding of a low-cost strapdown inertial measurement unit using vehicle model constraints for land vehicle applications, IEEE Trans. on Robotics and Automation, Vol. 17, No. 5, pp. 731-747, 2001.

[2] K. Mahata and T. Söderström, Improved estimation performance using known linear constraints, Automatica, Vol. 40, No. 8, pp. 1307-1318, 2004.

[3] S. Ko and R. R. Bitmead, State estimation for linear systems with state equality constraints, Automatica, Vol. 43, No. 8, pp. 1363-1368, 2007.

[4] D. Chu , T. Chen, and H. J. Marquez, Robust moving horizon state observer, IJC, Vol. 80, No. 10, pp. 1636-1650, 2007.

[5] J. B. Rawlings and D. Q. Mayne, Model predictive control: theory and design, LLC, Madison: Nob Hill Publishing, 2009.

[6] E. Chu, A. Keshavarz, D. Gorinevsky, and S. Boyd, Moving horizon estimation for staged QP problems, Proc. of IEEE CDC, pp. 3177-3182, Hawaii, 2012.
[7] M. Ge and E. C. Kerrigan, Relations between full information and Kalman-based estimation, Proc. of IEEE CDC, pp. 2041-2046, Las Vegas, 2016.

[8] C. V. Rao, J. B. Rawlings, and J. H. Lee, Constrained linear state estimation-a moving horizon approach, Automatica, Vol. 37, No. 10, pp. 1619-1628, 2001.

[9] H. Kong and S. Sukkarieh, Suboptimal receding horizon estimation via noise blocking, Automatica, Provisionally accepted, 2018.

[10] A. Alessandri, M. Baglietto, and G. Battistelli, Receding horizon estimation for discrete time linear systems, IEEE Trans. Autom. Control, Vol. 48, No. 3, pp. 473-478, 2003.

[11] D. Sui, T. A. Johansen, and L. Feng, Linear moving horizon estimation with pre-estimating observer, IEEE Trans. Autom. Control, Vol. 55, No. 10 , pp. 2363-2368, 2010.

[12] D. Sui and T. A. Johansen, Linear constrained moving horizon estimator with pre-estimating observer, Systems \& Control Letters, Vol. 67, pp. 40-45, 2014.

[13] Y. S. Shmaliy, S. Zhao, and C. K. Ahn, Unbiased finite impluse response filtering: an iterative alternative to Kalman filtering ignoring noise and initial conditions, IEEE Control Systems Magazine, Vol. 37, No. 5, pp. 70-89, 2017.

[14] C. K. Ahn, P. Shi, and M. V. Basin, Deadbeat dissipative FIR filtering, IEEE Trans. on Circuits and Systems I: Regular Papers, Vol. 63, No. 8 , pp. 1210-1221, 2016.

[15] H. Kong, G. C. Goodwin, and M. M. Seron, A revisit to inverse optimality of linear systems, IJC, Vol. 85, No. 10, pp. 1506-1514, 2012.

[16] H. Kong, G. C. Goodwin, and M. M. Seron, Predictive metamorphic control, Automatica, Vol. 49, No. 12, pp. 3670-3676, 2013.

[17] E. N. Hartley and J. M. Maciejowski, Designing output-feedback predictive controllers by reverse engineering existing LTI controllers, IEEE Trans. on Automatic Control, Vol. 58, No. 11, pp. 2934-2939, 2013.

[18] A. J. Laub, Matrix analysis for scientists and engineers, SIAM, 2005.

[19] R. R. Bitmead, M. R. Gevers, I. R. Petersen, and R. J. Kaye, Monotonicity and stabilizability properties of solutions of the Riccati difference equation, System \& Control Letters, Vol. 5, No. 5, pp. 309-315, 1985.

[20] S. W. Chan, G. C. Goodwin, and K. S. Sin, Convergence properties of the Riccati difference equation in optimal filtering of nonstabilizable systems, IEEE Trans. Autom. Control, Vol. 29, No. 2, pp. 110-118, 1984.

[21] D. L. Kleinman and M. Athans, The design of suboptimal linear time varying equations, IEEE Trans. Autom. Control, Vol. 13, No. 2, pp. $150-159,1968$. 